\title{
The effect of fish oil versus krill oil on markers of metabolic syndrome and the plasma metabolome; a pilot study
}

\author{
W. Jones ${ }^{1}$, D. Scott, J. K. Lodge ${ }^{2}$, M. A. Schmidt ${ }^{3}$ and I. G. Davies ${ }^{1}$ \\ ${ }^{1}$ Faculty of Education, Community and Leisure, Liverpool John Moores University, Liverpool, L17 6BD, UK, \\ ${ }^{2}$ School of Life Sciences, Northumbria University, Newcastle NE1 8ST and ${ }^{3}$ Sovaris Aerospace LLC, Boulder, \\ CO 80302, USA
}

The long-term cardiovascular (CV) benefits of $n$-3 polyunsaturated fatty acids are well established and, despite dietary advice, most people do not consume sufficient oily fish. Fish oil (FO) supplements are an alternative source and are already widely taken amongst the general population. Krill oil (KO) has been suggested to have greater health benefits which may be due to a superior bioavailability ${ }^{(1)}$. The metabolic syndrome increases CV risk and is associated with small, dense low density lipoprotein (sdLDL) and insulin resistance, and is more prevalent in men. The current study aimed to pilot the effects of a low dose of KO versus FO in a middle aged male population on markers of metabolic syndrome, traditional markers of CVD, insulin resistance, sdLDL, and the plasma metabolome.

Male participants $(n=18,35-65 \mathrm{y})$ were recruited and randomly assigned to receive either $0.3 \mathrm{~g}$ of EPA/DHA from KO or $0.5 \mathrm{~g}$ of EPA/DHA from FO in a single blind randomised, crossover trial; participants supplemented their diet with either FO or KO for 6 weeks followed by a 6 week washout period before changing to the alternative supplement. Baseline, midpoint and endpoint markers of metabolic syndrome were measured ${ }^{(2)}$ : anthropometrics, plasma triglycerides (TG), high density lipoprotein cholesterol (HDL-C), systolic and diastolic blood pressure, and plasma glucose. Additional measures included: total plasma cholesterol, LDL-C, small dense LDL-C (sdLDL), and measures of insulin resistance. Nontargeted LC/MS based metabolite profiling analysis (positive ionisation) on deproteinized plasma samples pre and post intervention was also performed.

Repeated measures ANOVA analysis investigated any differences within and between groups. Results showed no significant main effect between groups on any of the above measures. However, there was a significant interaction between intervention and time for sdLDL $(p=0.038)$ showing a reduction in sdLDL \% at midpoint (FO). Furthermore, plasma TG showed a weak negative correlation with the change in plasma triglyceride from baseline to endpoint ( $\mathrm{KO}, p=0.28$; $\mathrm{FO}, p=0.09)$. Pairwise comparisons of plasma metabolite profiles revealed only subtle changes to the metabolome with treatment; one species (unknown, mass 453.322) was discriminatory for KO treatment being significantly increased in intensity pre $v s$. post $(p<0.005)$ and three unknown species were discriminatory for FO treatment (pre $v s$. post $p<0.05$, two species up-regulated, one species down-regulated).

In conclusion, the current study shows $\mathrm{KO}$ and FO, at relatively low doses, have minimal effects on markers of metabolic syndrome; however, there is weak evidence that participants with higher plasma triglycerides reduce plasma TG, but to a greater extent with FO and FO had a transient but positive effect on sdLDL. This pilot study has limitations with a small sample size, and dietary compliance and the plasma metabolome are still under investigation. The study is in partial agreement with previous research with respect to the effect on plasma $\mathrm{TG}^{(3)}$; however, further dose response studies are warranted.

1. Bunea R, El Farrah K and Deutsch L (2004) $A M R$ 9, 420-428.

2. Alberti KG, Eckel RH, Grundy SM et al. (2009) Circ 120, 1640-1645.

3. Ulven SM, Kirkhus B, Lamglait A et al. (2011) Lipids 46(1): 37-46. 